\title{
Scalp metastasis of advanced gastric cancer
}

\author{
Hyeong Rae Ryu', \\ Da Woon Lee', \\ Hwan Jun Choi ${ }^{1}$, \\ Jun Hyuk Kim ${ }^{1}$, \\ Hyein $\mathrm{Ahn}^{2}$ \\ Departments of ${ }^{1}$ Plastic and \\ Reconstructive Surgery and ${ }^{2}$ Pathology, \\ Soonchunhyang University College of \\ Medicine, Cheonan, Korea
}

\begin{abstract}
Head and neck cutaneous metastasis of advanced gastric cancer is uncommon, and scalp metastasis is particularly rare. We present the case of a 60-year-old man who was diagnosed with cutaneous metastasis on the scalp originating from advanced gastric cancer. The patient was referred to the plastic surgery department for a scalp mass near the hairline. He had been diagnosed with advanced gastric cancer and undergone total gastrectomy and Roux esophagojejunostomy 3 years previously. The differential diagnosis for a single flesh-colored nodule on the scalp included benign tumors such as epidermal cyst or lipoma; therefore, the patient underwent excision and biopsy. In the operative field, the mass was found to be located in the frontalis muscle. The biopsy result showed that the mass was a metastatic lesion of advanced gastric cancer. Whole-body computed tomography revealed a gastric tumor with blood vessel infiltration, peritoneal carcinomatosis, liver metastasis, and multiple disseminated subcutaneous metastases. Although scalp metastasis originating from an internal organ is extremely rare, plastic surgeons should always consider a metastatic lesion in the differential diagnosis if a patient with a scalp lesion has a history of malignant cancer.
\end{abstract}

Keywords: Advanced gastric cancer / Neoplasm metastasis / Scalp

\section{INTRODUCTION}

Cutaneous metastasis of internal organ malignancies is uncommon, with a reported prevalence of $5 \%$ to $9 \%$. Cutaneous involvement of gastric cancer is particularly unusual and usually manifests as nonspecific nodules in the vicinity of the primary tumor [1].

Gastric cancer mainly metastasizes to the lymph nodes, liver, and peritoneum, and skin metastasis has been rarely reported [2]. Most cutaneous metastases present around the umbilicus, and rarely metastasize to skin distant from the primary site [3]. The scalp is a particularly unusual site of cutaneous metastasis.

\section{Correspondence: Hwan Jun Choi}

Department of Plastic and Reconstructive Surgery, Soonchunhyang University

Cheonan Hospital, Soonchunhyang University College of Medicine,

31 Suncheonhyang 6-gil, Dongnam-gu, Cheonan 31151, Korea

E-mail: iprskorea@gmail.com

This work was supported by the National Research Foundation of Korea (NRF) grant funded by the Korea government (MSIT) (2020R1A2C1100891), and was supported by Soonchunhyang University research fund.

Received March 31, 2021 / Revised April 10, 2021 / Accepted April 20, 2021
Brownstein and Helwig [4] reported that scalp metastases account for $4 \%$ of all skin metastases. Few cases have been reported to date [5]. The difficulty of detecting lesions in hair-bearing areas results in delayed diagnoses, contributing to a poor prognosis.

This report presents a case of scalp metastasis from advanced gastric cancer and a review of the related literature. In light of this case, clinicians should keep in mind the possibility of cutaneous metastasis if a skin lesion occurs in a patient with a history of malignancy.

\section{CASE REPORT}

A 60-year-old man presented with an asymptomatic scalp mass and was referred to the plastic and reconstructive surgery department. The lesion had been slowly growing for approximately 2 years, reaching a size of $1 \mathrm{~cm}$ in diameter at the time of the visit. On physical examination, the mass was found to be a single well-defined, fixed, skin-colored indurated nodule on 

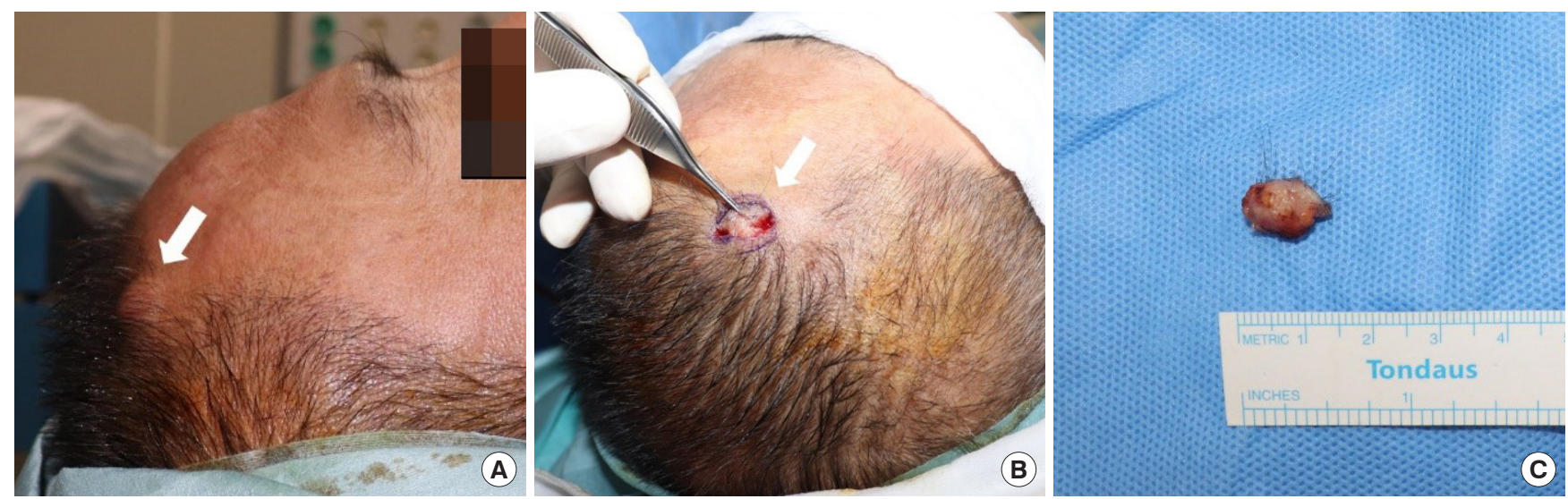

Fig. 1. A $1 \mathrm{~cm}$-sized nodule (white arrow) attached to the frontalis muscle. (A) Preoperative photograph. (B) Intraoperative photograph. (C) Photograph of the specimen.

the temporal scalp just above the hairline (Fig. 1A). The suspected diagnoses included epidermal cyst, lipoma, or other benign neoplasms, and a further differential diagnosis was deemed necessary. Excision and biopsy were performed for an accurate diagnosis and treatment. The incision line was designed with a 5-mm free margin from the mass. After dissecting the skin and soft tissue, an irregularly shaped mass with heterogeneous high vascularity attached to the frontalis muscle was observed (Fig. 1B and C). The mass was completely removed without any remnant tissue, and the skin was sutured. Microscopic findings of the scalp mass revealed tubular structures lined by mucin-secreting columnar epithelium in the dermis, consistent with metastatic adenocarcinoma (Fig. 2).

A thorough history was taken to identify the primary cancer of cutaneous metastasis. The patient had a history of advanced gastric cancer (T3N1M0, stage IIB) and undergone total gastrectomy and Roux esophagojejunostomy 3 years previously. Considering the biopsy findings and the patient's history, the metastatic adenocarcinoma was thought to have originated from advanced gastric cancer. As a cutaneous metastasis was found, an exploratory laparotomy was performed for re-staging. In the operative field, peritoneal seeding, pancreatic invasion, and celiac trunk invasion were observed. Since the tumor invaded adjacent structures and distant metastasis was found, the stage of advanced gastric cancer was finally determined as T4bN1M1 (stage IV). The patient then received combined chemotherapy treatment with leucovorin, fluorouracil, oxaliplatin, ramucirumab, and paclitaxel. During chemotherapy, a stricture of previous surgical site was found, and the patient underwent palliative surgery. Whole-body computed tomography revealed a gastric tumor with blood vessel infiltration, peritoneal carcinomatosis, liver metastasis, and multiple disseminated subcutaneous metastases. One year later, the patient died.

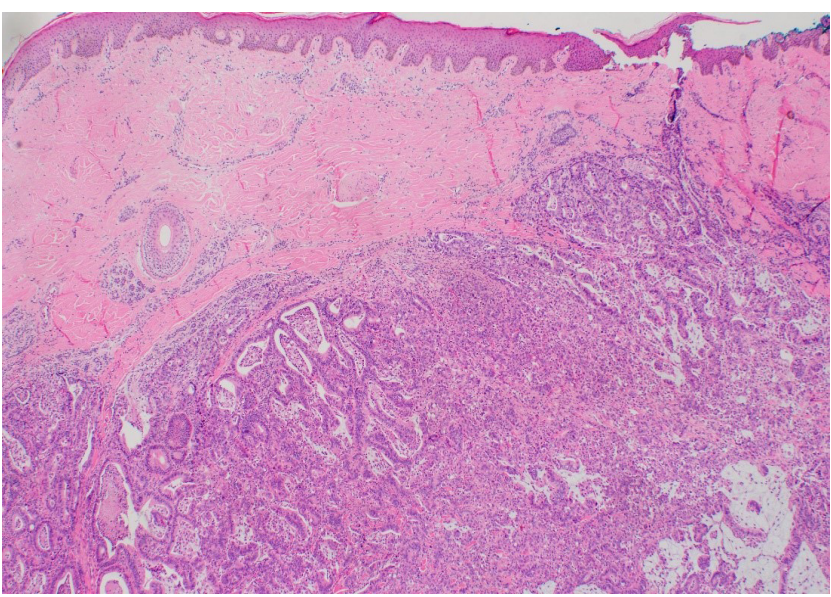

Fig. 2. Histopathologic examination showing skin metastasis originating from gastric adenocarcinoma $(\mathrm{H} \& \mathrm{E}, \times 40)$.

\section{DISCUSSION}

The prevalence of cutaneous metastasis of internal organ malignancies is about $5 \%$ to $9 \%$ [1]. Most cutaneous metastases involve direct or local metastases of breast cancer. Excluding breast cancer, cutaneous metastases of visceral organ malignancies were reported as occurring in fewer than $1 \%$ of cases [5]. Cutaneous metastasis takes place through one of two mechanisms of spread. In most cancers, it occurs through lymphatic metastasis, with each cancer having a preferred skin site of metastasis. Typically, lung cancer metastasizes to skin above the diaphragm, while colon cancer or genitourinary cancer tends to metastasize to skin below the diaphragm; this pattern suggests lymphatic metastasis. However, some cancers metastasize hematogenously. It is hypothesized that hematogenous metastasis occurs through a process in which tumor cells escape from the primary site, are intravasated into vessels, and then settle in sec- 
ondary organs [6]. Through this process, it is possible that metastatic cancer can occur randomly and far away. Some cases of randomly located cutaneous metastasis have been reported for other cancer types, such as renal cancer, suggesting hematogenous metastasis [5].

Gastric cancer mainly metastasizes to the lymph nodes, liver, and peritoneum. Cutaneous metastasis of gastric cancer is uncommon [2]. Most cutaneous metastases present around the umbilicus, and the proposed mechanisms include direct transperitoneal spread and spread via lymphatics near the obliterated umbilical vein [6]. Meanwhile, metastasis to the skin distant from the primary gastric cancer, which suggests hematogenous metastasis, is extremely rare [3]. A previous report showed that six out of seven gastric cancer metastases occurred in the umbilicus, abdomen and lower chest, and only one case occurred in the upper arm, which is distant from the stomach [5]. In addition, hematogenous metastasis is known to be associated with higher cancer-related mortality than lymphatic metastasis in gastric cancer, underscoring the need for awareness of this entity [7].

The incidence of tumors on the scalp is increasing compared to those occurring elsewhere on the skin. Only about $1 \%$ to $2 \%$ of all scalp tumors are malignant, accounting for up to $13 \%$ of all malignant skin tumors [8]. Cutaneous metastasis to the head and neck region can originate from some internal organ malignancies. The primary cancers that most often spread to the skin in the head and neck area are breast cancer, hematopoietic and lymphoid cancer, and lung cancer [3]. However, some primary cancers also rarely exhibit spread to the scalp. Lee et al. [9] reported a case of metastatic renal cell carcinoma in the head and neck area. To the best of our knowledge, only a few cases of cutaneous metastasis of gastric cancer in the head and neck area have been reported. In one case, a 91-year-old man presented an ulcerative plaque on his chin and was diagnosed with skin metastasis from gastric cancer. Further evaluation revealed numerous metastases in locations including the vessels, peritoneum, lung, and scattered subcutaneous sites [10]. In a small number of scalp metastasis cases, it has been reported that skin lesions can appear in the form of nodules, plaques, and alopecia on the scalp, but no specific clinical manifestation is associated with cutaneous metastasis. Another noteworthy point is that lesions in hair-bearing areas are difficult to detect, which leads to a delayed diagnosis, ultimately contributing to a poor prognosis. In our case, a patient with a history of advanced gastric cancer presented with a skin lesion on the scalp. It was first suspected to be a benign tumor, such as an epidermal cyst or lipoma, based on clinical findings. However, it was finally diagnosed as a cutaneous metastasis originating from primary gastric cancer. Since the location of cutaneous metastasis was distant and op- posite site of the primary cancer, hematogenous metastasis was suspected, rather than lymphatic metastasis. Cutaneous gastric cancer metastasis most often presents as Sister Mary Joseph nodules via direct or lymphatic spread; thus, our case is thought to be extremely rare.

The prognosis of advanced gastric cancer is known to be poor, with a 5-year survival rate of $3.1 \%$. The median survival of patients with unresectable advanced gastric cancer is $10-18$ months [11]. In addition, as mentioned above, cancer-related mortality in gastric cancer is higher in patients with hematogenous metastasis than in those with lymphatic metastasis [7]. Our patient died 1 year after the detection of distant metastasis despite chemotherapy and palliative surgery. In this context, our case is noteworthy from an educational standpoint, as it suggests that clinicians should pay close attention to abnormal skin lesions that occur even in areas far from the site of primary gastric cancer.

In conclusion, we experienced a rare case of cutaneous metastasis on the scalp originating from distant primary gastric cancer. Clinicians should always keep in mind that cutaneous metastasis is a possible diagnosis when skin lesions occur in patients with preexisting internal organ malignancies, as metastasis can occur to any site of the skin, including the scalp.

\section{NOTES}

\section{Conflict of interest}

No potential conflict of interest relevant to this article was reported.

\section{Ethical approval}

The study was approved by the Institutional Review Board of Soonchunhyang University Cheonan Hospital (IRB No. 202103-033) and performed in accordance with the principles of the Declaration of Helsinki. Written informed consent was obtained.

\section{Patient consent}

The patient provided written informed consent for the publication and the use of his images.

\section{ORCID}

Hyeong Rae Ryu https://orcid.org/0000-0003-0962-2407

Da Woon Lee https://orcid.org/0000-0002-0266-8480

Hwan Jun Choi https://orcid.org/0000-0002-0752-0389 Jun Hyuk Kim https://orcid.org/0000-0002-0266-8480 Hyein Ahn 


\section{Author contribution}

Conceptualization: HJC. Resources: HA. Supervision: DWL, JHK. Writing - original draft: HRR. Writing - review \& editing: HRR, HJC, DWL.

\section{REFERENCES}

1. Lookingbill DP, Spangler N, Sexton FM. Skin involvement as the presenting sign of internal carcinoma: a retrospective study of 7316 cancer patients. J Am Acad Dermatol 1990;22:19-26.

2. Namikawa T, Munekage E, Munekage M, Maeda H, Yatabe T, Kitagawa $\mathrm{H}$, et al. Subcutaneous metastasis arising from gastric cancer: a case report. Mol Clin Oncol 2017;6:515-6.

3. Pereira WA, Humaire CR, Silva CS, Fernandes LH. Sister Mary Joseph's nodule: a sign of internal malignancy. An Bras Dermatol 2011;86(4 Suppl 1):S118-20.

4. Brownstein MH, Helwig EB. Patterns of cutaneous metastases Arch Dermatol 1972;105:862-8.

5. DU C, Hong R, Liu Y, Wang J, Zhang H, Yu X. Scalp metastasis from gastric cancer: a case report and literature review. Oncol Lett 2015;9:641-4.

6. Cohen DC. A man with an umbilical ulcer. Medscape J Med 2008;10:11.

7. Zhong J, Chen Y, Wang LJ. Emerging molecular basis of hematogenous metastasis in gastric cancer. World J Gastroenterol 2016;22:2434-40.

8. Suk S, Shin HW, Yoon KC, Kim J. Aggressive cutaneous squamous cell carcinoma of the scalp. Arch Craniofac Surg 2020;21: 363-7.

9. Lee DW, Ryu HR, Kim JH, Choi HJ, Ahn H. Isolated temporalis muscle metastasis of renal cell carcinoma. Arch Craniofac Surg 2021;22:66-70.

10. Kirchberger MC. Unusual presentation of a cutaneous metastasis in the face arising from gastric cancer: a case report. SAGE Open Med Case Rep 2018;6:2050313X18795080.

11. Leiting JL, Grotz TE. Advancements and challenges in treating advanced gastric cancer in the West. World J Gastrointest Oncol 2019;11:652-64. 